\title{
ALGEBRAIC STATISTICS FOR A DIRECTED RANDOM GRAPH MODEL WITH RECIPROCATION
}

\author{
SONJA PETROVIĆ, ALESSANDRO RINALDO, AND STEPHEN E. FIENBERG
}

\begin{abstract}
The $p_{1}$ model is a directed random graph model used to describe dyadic interactions in a social network in terms of effects due to differential attraction (popularity) and expansiveness, as well as an additional effect due to reciprocation. In this article we carry out an algebraic statistics analysis of this model. We show that the $p_{1}$ model is a toric model specified by a multi-homogeneous ideal. We conduct an extensive study of the Markov bases for $p_{1}$ models that incorporate explicitly the constraint arising from multi-homogeneity. Our results are directly relevant to the estimation and conditional goodness-of-fit testing problems in $p_{1}$ models.
\end{abstract}

\section{INTRODUCTION}

The study of random graphs is an active area of research in many fields, including mathematics, probability, biology and social sciences. In the social network literature, the nodes of the network represent population units and the edges represent relationships among individuals. Thus, prescribing a probability distribution over a network is essentially a way of encoding the dynamic of interactions and behavior among individuals in a given population. For a review, see Goldenberg et al. [12].

One of the earlier and most popular statistical models for social networks is the $p_{1}$ model of Holland and Leinhardt, described in their 1981 seminal paper [15]. In a $p_{1}$ model the network is represented as a digraph in which, for every pair of nodes $\{i, j\}$, or dyad, one can observe one of four possible dyadic configurations: a directed edge from $i$ to $j$, a directed edge from $j$ to $i$, two directed edges, or no edges between $i$ and $j$. Each dyad is in any of these configurations independently of all the other dyads. Quoting from [15], the probability distribution over the entire network depends on various parameters quantifying the "attractiveness" and "productivity" of the individual nodes, the "strength of reciprocity" of each dyad and the network "density," or overall tendency to have edges. See the next section for details.

Despite its simplicity and interpretability, and despite being a special case of the broader and well studied class of log-linear models, e.g., see [10, 11], the $p_{1}$ model poses extraordinary challenges of both theoretical and practical nature. Indeed, first and foremost, the number of network parameters depends on the size of the network, so that, as the population size grows, the model complexity also increases. This remarkable feature renders the $p_{1}$ model, as well as virtually any other network model, unlike traditional statistical models (including log-linear models), whose complexity is fixed and independent of the sample size. Second,

Stephen E. Fienberg and Alessandro Rinaldo supported in part by NSF Grant DMS-0631589 to Carnegie Mellon University. 
as assumed by Holland and Leinhardt in their original paper [15], and as is customary in social network analysis, statistical inference for the $p_{1}$ model is typically made on the basis of one realization of the network, which amounts to one observation per dyad. Because of the unavoidable sparsity and low information content of the observable data, and because of the dependence of the model complexity on the sample size, classical statistical procedures for estimation and goodness-of-fit testing in $p_{1}$ models may be inadequate and exhibit properties that are poorly understood.

In this article, we revisit the Holland-Leinhardt $p_{1}$ model using the tools and language of algebraic geometry, as is customary in the maturing field of algebraic statistics. See, e.g., [7], [17] and [8]. For the class of $p_{1}$ models at hand, we identify the probabilities of each of the $2 n(n-1)$ possible dyadic configurations with the indeterminates of a multi-homogeneous toric ideal. Our first goal is to investigate the Markov bases for $p_{1}$ by computing a minimal generating set of this ideal first and then by removing basis elements that violate the condition of one observation per dyad. To our knowledge, this is the first time in the Markov bases literature that sampling constraints of this form, known in statistics as product Multinomial sampling schemes, have been directly incorporated in the study of Markov bases. Our results prescribe a set of rules for creating Markov moves that are applicable to network data and offer significant efficiency improvements over existing methods for generating Markov bases. Our second goal is to study the toric variety associated to the $p_{1}$ ideal and to relate its geometric properties with the conditions for existence of the maximum likelihood estimator of the dyadic probabilities.

The paper is organized as follows. In Section 2 we describe in detail the $p_{1}$ model, its statistical properties and the statistical tasks of estimation and testing that motivate our work. In particular, we consider three different flavors of the $p_{1}$ model, of increasing complexity. In Section 3 we embark on an exhaustive study of the Markov bases for $p_{1}$ models. As we mentioned above, the constraint that there is only one observation per dyad makes our analysis unusually difficult.

\section{The Holland-LeindhardT $p_{1}$ MODEL}

We consider a directed graph on the set of $n$ nodes. The nodes correspond to units in a network, such as individuals, and the edges correspond to links between the units. We focus on dyadic pairings and keep track of whether node $i$ sends an edge to $j$, or vice versa, or none, or both. Let $p_{i j}(1,0)$ be the probability of $i$ sending an edge toward $j$, and let $p_{i j}(0,1)$ the probability of $j$ sending an edge toward $i$ (thus, 1 denotes the outgoing side of the edge). Further, $p_{i j}(1,1)$ is the probability of $i$ sending an edge to $j$ and $j$ sending an edge to $i$, while $p_{i j}(0,0)$ is the probability that there is no edge between $i$ and $j$. Thus,

$$
p_{i j}(0,0)+p_{i j}(1,0)+p_{i j}(0,1)+p_{i j}(1,1)=1,
$$

for each of the $\left(\begin{array}{l}n \\ 2\end{array}\right)$ pairs of nodes $\{i, j\}$. The Holland-Leinhardt $p_{1}$ model is given as follows (see [15]):

$$
\begin{aligned}
& \log p_{i j}(0,0)=\lambda_{i j} \\
& \log p_{i j}(1,0)=\lambda_{i j}+\alpha_{i}+\beta_{j}+\theta \\
& \log p_{i j}(0,1)=\lambda_{i j}+\alpha_{j}+\beta_{i}+\theta \\
& \log p_{i j}(1,1)=\lambda_{i j}+\alpha_{i}+\beta_{j}+\alpha_{j}+\beta_{i}+2 \theta+\rho_{i j}
\end{aligned}
$$


The real-valued variables $\theta, \alpha_{i}, \beta_{i}, \rho_{i j}$ and $\lambda_{i j}$ for all $i<j$ are the model parameters. The parameter $\alpha_{i}$ controls the effect of an outgoing edge from $i$, the parameter $\beta_{j}$ the effect of an incoming edge into $j$, and $\rho_{i j}$ the added effect of reciprocated edges (in both directions). The "density" parameter $\theta$ quantifies the overall propensity of the network to have edges. Finally, $\lambda_{i j}$ is just a normalizing constant to ensure that (2.1) holds for each each dyad $\{i, j\}$. See the original paper on the $p_{1}$ model [15] for an extensive interpretation of the model parameters.

We take note here of a basic, yet a rather fundamental feature of our settings that apply to $p_{1}$ models as well as to many other statistical models for networks: data become available in the form of one observed network. Even though for each pair of nodes $\{i, j\}$, the four probabilities $p_{i j}(\bullet, \bullet)$ are strictly positive according to the defining equations $(2.2)$, each dyad can be observed in one and only one of the 4 possible states. Thus, despite the richness and complexity of $p_{1}$ models, their statistical analysis is severely limited by the disproportionally small information content in the data. This fact makes $p_{1}$ and, more generally, network models challenging, and it greatly affects our analysis, as we will show.

We study the following special cases of the general $p_{1}$ structure:

(1) $\rho_{i j}=0$ : no reciprocal effect.

(2) $\rho_{i j}=\rho$ : constant reciprocation.

(3) $\rho_{i j}=\rho+\rho_{i}+\rho_{j}$ : edge-dependent reciprocation.

For a network on $n$ nodes, we represent the vector of $2 n(n-1)$ dyad probabilities as $p=$ $\left(p_{12}, p_{13}, \ldots, p_{n-1, n}\right) \in \mathbb{R}^{2 n(n-1)}$, where, for each $i<j, p_{i j}=\left(p_{i j}(0,0), p_{i j}(1,0), p_{i j}(0,1), p_{i j}(1,1)\right) \in$ $\Delta_{3}$, with $\Delta_{3}$ the standard simplex in $\mathbb{R}^{4}$.

As usual in algebraic statistics, the $p_{1}$ model is defined to be the set of all candidate probability distributions that satisfy the Holland-Leinhardt equations (2.2). By definition, the $p_{1}$ model is $\log$-linear; that is, the set of candidate probabilities $p$ is such that $\log p$ is in the linear space spanned by the rows of a matrix $A$, which is often called the design matrix of the model. Indeed, the design matrix encodes a homomorphism between two polynomial rings: $\varphi_{n}: \mathbb{C}\left[p_{i j}(a, b)\right] \rightarrow \mathbb{C}\left[\lambda_{i j}, \alpha_{i}, \beta_{i}, \theta, \rho_{i j}\right]$, with $i<j \in\{1 \ldots n\}$ and $a, b \in\{0,1\}$, induced by

$$
p_{i j}(a, b) \mapsto \lambda_{i j} \alpha_{i}^{a} \alpha_{j}^{b} \beta_{i}^{b} \beta_{j}^{a} \theta^{a+b} \rho_{i j}^{\min (a, b)}
$$

where $a, b \in\{0,1\}$, and we consider parameters $\lambda_{i j}, \alpha_{i}, \beta_{i}, \rho_{i j}$ and $\theta$ for $i, j \in\{1, \ldots, n\}$ as unknowns. The design matrix $A$ simply describes the action of $\varphi_{n}$ on the probabilities $p_{i j}(\bullet, \bullet)$. The columns of $A$ are indexed by $p_{i j}$ 's and its rows by the model parameters. The entries of the design matrix are either 0 or 1 ; there is a 1 in the $(r, c)$-entry of the matrix if the parameter indexing row $r$ appears in the image of the $p_{i j}$ indexing the column $c$. For example, in the case $\rho_{i j}=0$, the matrix $A$ is of size $(2 n) \times\left(3\left(\begin{array}{l}n \\ 2\end{array}\right)\right)$. We will create the design matrices in a systematic way: the rows will always be indexed by $\lambda_{i j}, \alpha_{1}, \ldots, \alpha_{n}, \beta_{1}, \ldots, \beta_{n}$, $\theta, \rho_{i j}$ lexicographically in that order. The columns will be ordered in the following way: first fix $i$ and $j$ in the natural lexicographic ordering; then, within each set, vary the edge directions in this order: $(0,0),(1,0),(0,1),(1,1)$. Examples can be found in section 3.2 . Furthermore, it is easy to see that the design matrix for the network on $n$ nodes will consist of several copies of the 2-node matrix, placed as a submatrix of in those rows and columns corresponding to all 2-node subnetworks. 
Let $\zeta=\left(\zeta_{1}, \ldots, \zeta_{d}\right)$ denote the vector of model parameters, whose dimension $d$ depends on the type of restrictions on the $p_{1}$. Then, using the design matrix $A$, one can readily verify that the Holland-Leinhardt equations have the $\log$-linear representation $\log p=A^{\top} \zeta$, or, equivalently, letting $a_{k, l}$ and $p_{k}$ be the $(k, l)$ entry of $A$ and the $k$-th element of $p$, respectively,

$$
p_{k}=\prod_{l=1}^{d}\left(a_{k, l}\right)^{\zeta_{l}} .
$$

2.1. Algebraic statistical challenges in $p_{1}$ models. To provide some context and motivation for our analysis, we now briefly review some fundamental statistical tasks for the analysis of $p_{1}$ models. We point out that these problems still remain in part unsolved.

Denote by $M_{A}$ the $p_{1}$ model, that is, the set of points satisfying the Holland-Leinhardt equations $(2.2)$. Notice that $M_{A}$ is a relatively open set in the positive orthant of $\mathbb{R}^{2 n(n-1)}$ of dimension $\operatorname{rank}(A)$. Let $\mathcal{X}_{n} \subset \mathbb{R}^{2 n(n-1)}$ be the sample space, that is, the set of all observable networks on $n$ nodes. Notice that $\left|\mathcal{X}_{n}\right|=4^{n(n-1)}$. We will write every point $x$ in the sample space $\mathcal{X}$ as $x=\left(x_{12}, x_{13}, \ldots, x_{n-1, n}\right)$, where each of the $\left(\begin{array}{l}n \\ 2\end{array}\right)$ subvectors $x_{i j}$ is a vertex of $\Delta_{3}$. Probabilistically, each $x_{i j}$ is the realization of a random vector in $\mathbb{R}^{4}$ having multinomial distribution with size 1 and class probabilities $p_{i j}^{0} \in \Delta_{3}$, where $p^{0}=\left(p_{12}^{0}, p_{13}^{0}, \ldots, p_{n-1, n}^{0}\right)$ is an unknown vector in $M_{A}$. Furthermore, 2.2 implies the multinomial vectors representing the dyad configurations are mutually independent.

Once a network $x \in \mathcal{X}_{n}$ has been observed, statisticians are interested in the following interrelated fundamental problems:

1) estimation problem: to estimate the unknown vector $p^{0} \in M_{A}$;

2) goodness-of-fit problem: to test whether the $p_{1}$ model $M_{A}$ can be considered appropriate, i.e. whether the estimate produced in part (a) is a good fit to the observed data $x$.

To tackle the first problem, the most commonly used method is maximum likelihood estimation. Specifically, for a fixed point $x \in \mathcal{X}_{n}$, the likelihood function $\ell: M_{A} \rightarrow[0,1]$ given by

$$
\ell_{x}(p)=\prod_{i<j}\left(\prod_{k=1}^{4} p_{i j}(k)^{x_{i j}(k)}\right)
$$

returns the probability of observing the network $x$ as a function of the multinomial probabilities $p \in M_{A}$. The maximum likelihood estimator, or MLE, of $p^{0}$ is

$$
\hat{p}=\operatorname{argmax}_{p \in M_{A}} \ell_{x}(p),
$$

i.e. the vector of multinomial probabilities in $M_{A}$ that makes $x$ the most likely network to have been observed. See [18] for more details on maximum likelihood for this model.

While the estimation problem is relatively well constrained, the problem of testing for goodness of fit has a much broader scope, as it entails testing whether the assumption that $p^{0} \in M_{A}$ is itself appropriate. A typical goodness-of-fit testing proceeds through the following steps:

1) Compute the MLE $\hat{p}$ as defined in 2.3 .

2) Compute the goodness-of-fit statistic $G F(x)$. This quantity measures how close the MLE is to the observed network, or, using a statistical jargon, "how well the model $M_{A}$ fits the 
data $x . "$ Among the most commonly used statistics are Pearson's $\chi^{2}$ and the likelihood ratio statistic:

respectively.

$$
\sum_{i<k} \sum_{k=1}^{4} \frac{\left(\hat{p}_{i j}(k)-x_{i j}(k)\right)^{2}}{\hat{p}_{i j}^{2}(k)} \text { and } \sum_{i<k} \sum_{k=1}^{4} x_{i j}(k) \log \left(\frac{x_{i j}(k)}{\hat{p}_{i j}(k)}\right)
$$

3) Reject the assumption that $p^{0} \in M_{A}$ if the goodness-of-fit statistic used in step (2) is statistically large.

Step (3) above is clearly the most delicate, as there is no generally valid recipe for deciding when $G F(x)$ is too large, one of the reasons being that the goodness-of-fit statistic is itself a random variable. In many applications, it is customary to declare a model a poor fit if $G F(x)$ is greater than a given deterministic threshold (depending on the size of the network and the number $d$ of model parameters), which is obtained based on asymptotic approximations. Despite their widespread use, for the present problem these approximations have no theoretical justification (see, e.g., [13]) and can, in fact, be very poor.

Markov bases provide an alternative, non-asymptotic approach to performing goodnessof-fit tests and model selection. Their use has gained some momentum and popularity in recent years. Let $t=A x$ denote the vector of margins, or sufficient statistics, corresponding to the observed network. Let $\mathcal{T}_{t}=\left\{x^{\prime} \in \mathcal{X}_{n}: A x^{\prime}=t\right\} \subset \mathcal{X}_{n}$ be the fiber of $t$. From statistical theory (see, e.g., [2]), all the networks in $\mathcal{X}_{n}$ belonging to the same fiber will produce the same MLE. Thus, from the inferential standpoint, all the networks in the same fiber are equivalent. Based on this, if $x$ is the observed network and $t$ the associated sufficient statistic, one can decide whether the model $M_{A}$ provides a good fit if the quantity

$$
\alpha_{x}=\frac{\left|\left\{x^{\prime} \in \mathcal{T}_{t}: G F\left(x^{\prime}\right)>G F(x)\right\}\right|}{\left|\mathcal{T}_{t}\right|}
$$

is statistically large. Notice that $\alpha_{x}$ is the fraction of networks in the fiber at $t$ whose goodness-of-fit statistic value is larger than the value at the true network $x$. Heuristically, if $\alpha_{x}$ is large, i.e. closer to 1 , then $\hat{p}$ is closer to the observed network than most of the other points in the same fiber, thus implying that the model fits really well.

Despite its appeal, this methodology is rarely feasible due to the high computational costs of determining the fiber. Thus, rather than computing the fiber exactly, one can attempt to estimate $\alpha_{x}$ by performing a sufficiently long random walk over $\mathcal{F}_{t}$, as follows. A Markov basis for $M_{A}$ is set of vectors $\mathcal{M}=\left\{f_{1}, \ldots, f_{M}\right\} \subset \mathbb{Z}^{2 n(n-1)}$ such that

(i) $\mathcal{M} \subset \operatorname{kernel}(A)$;

(ii) for each observable margin $t$ and each $x, x^{\prime} \in \mathcal{T}_{t}$, there exists a sequence $\left(\epsilon_{1}, f_{i_{1}}\right), \ldots,\left(\epsilon_{N}, f_{i_{N}}\right)$ (with $N$ depending on both $x$ and $x^{\prime}$ ), such that $\epsilon_{j} \in\{-1,1\}$ for all $j$ and

$$
x^{\prime}=x+\sum_{j=1}^{N} \epsilon_{j} f_{i_{j}}, \quad x+\sum_{j=1}^{a} \epsilon_{j} f_{i_{j}} \in \mathcal{X}_{n} \text { for all } a=1, \ldots, N .
$$

Notice that Markov bases are not unique. If a Markov basis is available, then, for any observable network $x$ with sufficient statistics $t=A x$, it is possible to estimate $\alpha_{x}$ by walking at random inside the fiber $\mathcal{T}_{t}$ according to the following algorithm:

(1) set $\mathrm{k}=0$ and set $x^{\text {old }}=x$; 
(2) at every step, starting from the current position $x^{\text {old }}$,

(a) randomly choose a vector $f \in \mathcal{M}$ and a number $\epsilon \in\{-1,1\}$;

(b) if $x^{\text {old }}+\epsilon f \in \mathcal{X}_{n}$, move to $x^{\text {new }}=x^{\text {old }}+\epsilon f \in \mathcal{X}_{n}$, otherwise stay at $x^{\text {new }}=x^{\text {old }}$;

(3) if $G F\left(x^{\text {new }}\right)$ is larger than $G F(x)$ set $\mathrm{k}=\mathrm{k}+1$;

(4) repeat the steps (2) and (3) $K$ times.

Provided that the algorithm is run for a sufficiently long time (i.e. $K$ is large enough), the number $k / K$ is an accurate estimate of $\alpha_{x}$. See [7] for details and variants of the basic Markov basis algorithm described above.

\section{MARKOV BASES OF $p_{1}$ MODELS}

In this section we study the properties of Markov bases for the three versions of the $p_{1}$ model described in section 2 .

Our analysis presents certain aspects that set it apart from most of the existing literature on Markov bases. Indeed, the traditional algebraic geometry machinery generates Markov bases that are "universal", in the sense of depending only on the design matrix $A$, and not the sample space and its properties. The reason for this is that Markov bases are obtained as generating sets toric ideals, and thus they cut out a complex toric variety; while the model itself lies in the positive real part of the variety. As a result, Markov bases tend to be very large even for design matrices of moderate size. In contrast, as we noted above, the sample space for network data is very highly constrained, since each dyad can only be observed in one and only one of the four possible configurations. Consequently, many of the basis elements of any Markov bases are not applicable, since they violate this basic constraint of the data. Thus, once we find the Markov bases, we still need to be careful in identifying what elements are relevant to our data and in removing the moves that are not applicable. To our knowledge, this is the first time this issue has been addressed in the Markov bases literature.

On the other hand, we are able to decompose every Markov basis element using certain "basic" moves (Theorem 3.22), which are, as we will see, statistically meaningful by definition. The key idea is to decompose the toric ideal of the $p_{1}$ model using ideals which are known and easier to understand. Namely, ignoring the normalizing constants $\lambda_{i j}$ reveals a connection between $p_{1}$ models and toric varieties which are associated to certain graphs. These have been studied by the commutative algebra and algebraic geometry community, specifically Villarreal [20] and Ohsugi and Hibi [5]. We will use this connection to explain the form of Markov bases of $p_{1}$ models. In terms of ideal generators for the $p_{1}$ model, reintroducing the normalizing constants does add another level of difficulty. However, in terms of moves on the network, we can avoid this difficulty by exhibiting the decomposition of the moves (although inapplicable in terms of ideal generators) using well-understood binomials arising from graphs. This approach reduces the complexity and size of Markov moves. In addition, it allows us to bypass the study of those basis elements are not applicable due to the constraints described above.

3.1. The toric ideal of the $p_{1}$ model. Recall that the model consists of the points of the probability simplex that are in the row space of the design matrix. It follows that our model 
is, in fact, the positive part of the variety that is (the closure of) the image of the map $\varphi_{n}$. For more discussion on the geometry of the model, see [18].

To understand the model, we ask for all the polynomial equations that vanish on all of the points of the model; this set of equations is the defining ideal of the variety. In the case of log-linear models, the ideal is a toric ideal (see [19] for a standard reference). It can be computed from the kernel of the design matrix $M$ :

$$
I_{M}=\left(p^{u}-p^{v}: u-v \in \operatorname{kernel}(M)\right) \text {, }
$$

where $p^{u}$ denotes a monomial. Any generating set of the defining ideal $I_{M}$ gives a Markov basis for our model. This is the Fundamental Theorem of Markov Bases (see [7], or [8], Theorem 1.3.6). It describes the relations among the $p_{i j}(\bullet, \bullet)$, and can be used for a random walk in any given fiber consisting of the points with the same minimal sufficient statistics, and thus to compute the exact distribution of the model for goodness-of-fit purposes. Note that the sufficient statistics, in this case, are the in- and out- degree distributions of the nodes.

In order to enumerate all networks with the same degree distributions, one might want to use a Gröbner basis instead. A Gröbner basis is a generating set of the ideal, usually nonminimal, with some special structure desirable to have for computations. It is guaranteed to connect all points in a given fiber; every Gröbner basis is a Markov basis. A Gröbner basis can be made unique by requiring it to be reduced. There are finitely many reduced Gröbner bases, and the union of all of them is contained in the set of primitive binomials, called the Graver basis of the ideal. The Graver basis is generally very hard to compute, but sometimes easier to describe algebraically, and its structure naturally implies constraints on the structure of the minimal Markov moves.

Our first goal is to understand the structure of these Markov bases for the three cases of the $p_{1}$ model. Even though their size grows rapidly as we increase the number of nodes, there is quite a lot of structure in these generating sets. In what follows, we will first illustrate this structure on some small networks.

Let us first fix some notation for the remainder of the paper. Since there are three cases of the $p_{1}$ model, we need three different names for the design matrices of the $n$-node network. The design matrix depends on the choice of $n$ and $\rho_{i j}$ :

(1) For the case $\rho_{i j}=0$, when the reciprocal effect is zero, the design matrix for the $n$-node network will be denoted by $\mathcal{Z}_{n}$.

(2) For the case of constant reciprocation, i.e. $\rho_{i j}=\rho$, the $n$-node network matrix will be denoted by $\mathcal{C}_{n}$.

(3) When reciprocation is edge-dependent, i.e. $\rho_{i j}=\rho+\rho_{i}+\rho_{j}$, the design matrix will be denoted by $\mathcal{E}_{n}$.

\subsection{Markov bases of small networks.}

3.2.1. Case I: no reciprocation. $\left(\rho_{i j}=0\right)$

This is clearly a special case of $\rho_{i j}=\rho$, but we treat it here separately. We will see that, algebraically, it is interesting in its own right. 
Let's start with the simplest nontrivial example: $n=2$. The design matrix

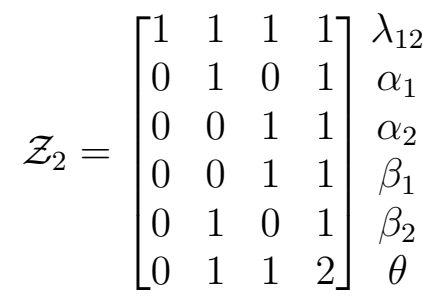

encodes the parametrization $\varphi_{2}$ of the variety. Its rows are indexed by parameters as indicated, while the columns are indexed by $p_{12}(0,0), p_{12}(1,0), p_{12}(0,1), p_{12}(1,1)$. The ideal $I_{\mathcal{Z}_{2}}=\left(p_{12}(1,0) p_{12}(0,1)-p_{12}(1,1) p_{12}(0,0)\right)$ is the principal ideal generated by one quadric, and thus this single binomial is a Markov basis, and also a Gröbner basis with respect to any term order. This can be verified by hand, or using software such as 4ti2 [1].

Remark 3.1 (From binomials to moves). In general, we can translate binomials to moves in the following way: we will remove all edges that are represented by the $p_{i j}$ 's in the negative monomial, and add all edges represented by the $p_{i j}$ 's in the positive monomial. Note that if $p_{i j}(0,0)$ occurs in either, it has no effect: it says to remove or add the "no-edge", so nothing needs to be done. However, there is a reason why $p_{i j}(0,0)$ 's show up in the ideal: the structure of $\mathcal{Z}_{n}$ for any $n$ requires that each binomial in the ideal is homogeneous with respect to the pair $\{i, j\}$. Here, for example, since the positive monomial is of degree two, the negative monomial has $p_{12}(0,0)$ attached to it to ensure it also is of degree two. Thus, the generator of $I_{\mathcal{Z}_{2}}$ represents the following Markov move:

Delete the bidirected edge between 1 and 2 , and replace it by two edges: $(1,2)$ and $(2,1)$.

However, if we would like to require at most one edge per dyad, then this binomial is meaningless and there aren't really any allowable Markov moves. Logically, the case of no reciprocation somehow contradicts this requirement, since if $\rho_{i j}=0$, we always get that a bi-directed edge between two nodes is valued the same as two edges between them. Thus the assumption of only one edge per dyad makes this problem so much more complicated, as relations like this one for any dyad in an $n$-node network will appear in the generating sets of the ideal $I_{\mathcal{Z}_{n}}$, but we will never want to use them.

Next, let $n=3$. The toric ideal $I_{\mathcal{Z}_{3}}$ is minimally generated by the following set of binomials: $p_{23}(0,1) p_{23}(1,0)-p_{23}(1,1) p_{23}(0,0), p_{13}(0,1) p_{13}(1,0)-p_{13}(1,1) p_{13}(0,0), p_{12}(0,1) p_{12}(1,0)-$ $p_{12}(1,1) p_{12}(0,0), p_{12}(0,1) p_{13}(1,0) p_{23}(0,1)-p_{12}(1,0) p_{13}(0,1) p_{23}(1,0)$. It is interesting to note that the first 3 generators are precisely the binomials from $I_{\mathcal{Z}_{2}}$ for the three dyads $\{1,2\}$, $\{1,3\}$, and $\{2,3\}$. The only statistically meaningful generator is the cubic. It represents the following move:

Replace the edges $(1,2),(2,3),(3,1)$ by $(2,1),(3,2),(1,3)$.

Graphically, it represents the three-cycle oriented two different ways: the positive monomial represents the cycle $(1,3,2,1)$, while the negative monomial represents its inverse, $(1,2,3,1)$, as depicted in figure 3.1 .

Suppose now that $n=4$. A minimal generating set for the ideal $I_{\mathcal{Z}_{4}}$ consists of 151 binomials: 6 quadrics, 4 cubics, 93 quartics and 48 quintics. Some of these violate the 


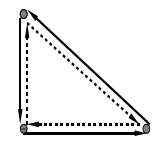

FiguRE 3.1. A degree-three move on 3 nodes: dashed edges are replaced by full edges.

requirement that each dyad can be observed in only one state. As it is impractical to write all of these binomials down, we will list just a few of those that are statistically meaningful (i.e. respect the requirement of at most one edge per dyad at any time). As expected, the quadrics and the cubics are simply the generators of $I_{\mathcal{Z}_{3}}$ for the four 3-node subnetworks of the four-node network. As we've seen, the quadrics are not of interest. The cubics represent the three-cycles. Here is a list of sample quartics, written in binomial form: $p_{12}(1,1) p_{34}(1,1) p_{23}(0,0) p_{14}(0,0)-p_{12}(0,0) p_{34}(0,0) p_{23}(1,1) p_{14}(1,1)$, $p_{23}(1,1) p_{14}(1,1) p_{13}(0,0) p_{24}(0,0)-p_{23}(1,0) p_{14}(1,0) p_{13}(0,1) p_{24}(0,1)$, $p_{23}(1,1) p_{14}(1,1) p_{12}(0,0) p_{34}(0,0)-p_{12}(1,0) p_{23}(1,0) p_{34}(1,0) p_{14}(0,1)$, $p_{12}(0,0) p_{23}(1,1) p_{34}(0,1) p_{14}(1,0)-p_{12}(1,0) p_{23}(1,0) p_{34}(1,1) p_{14}(0,0)$.

Finally, we list some representative quintics:

$p_{12}(0,0) p_{23}(1,1) p_{34}(0,1) p_{14}(0,1) p_{24}(1,0)-p_{12}(0,1) p_{23}(1,0) p_{34}(1,1) p_{14}(0,0) p_{24}(0,1), p_{12}(1,0) p_{23}(1,0) p_{14}(0,0$ $p_{12}(0,1) p_{23}(1,1) p_{14}(1,0) p_{13}(1,0) p_{24}(0,0)$.

This set of Markov moves is quite more complex then the 10 moves originally described by Holland and Leinhardt for the 4-node case. We will postpone any further analysis of these binomials until the next section. For now, let us note that all of them preserve the in- and out- degree distributions of the nodes in the network. After we study the other two cases for $\rho_{i j}$, we will see a recurring underlying set of moves which can be used to understand the ideals.

\subsubsection{Case II: constant reciprocation. $\left(\rho_{i j}=\rho\right)$}

Now we introduce one more row to the zero- $\rho$ design matrix $\mathcal{Z}_{n}$ to obtain the constant- $\rho$ matrix $\mathcal{C}_{n}$. Namely, this row represents the constant $\rho$ added to those columns indexed by $p_{i j}(1,1)$ for all $i, j \in[n]$. It is filled with the pattern $0,0,0,1$ repeated as many times as necessary. For example, the design matrix for the 2-node network is as follows:

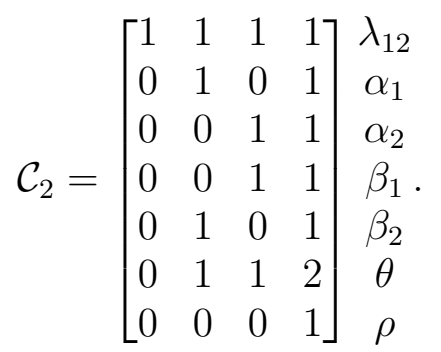

In this case the ideal is empty (there is nothing in the kernel of $\mathcal{C}_{2}$ ), which is expected since the only relation in the case of $\rho_{i j}=0$ required that there is no reciprocation. Here, the bidirected edge is valued differently then the two single edges in a dyad; this is the meaning of the last row of the design matrix. 
For the 3-node network, the Markov move consist only of the cubic from the case $\rho_{i j}=0$ : $p_{12}(0,1) p_{13}(1,0) p_{23}(0,1)-p_{12}(1,0) p_{13}(0,1) p_{23}(1,0)$. On a side note, even the Graver basis consists only of this move and 15 other non-applicable moves (those which ignore the singleedged dyad assumption).

Let $n=4$. The software 4 ti2 outputs a minimal generating set of the ideal $I_{\mathcal{C}_{4}}$ consisting of: 4 cubics, 57 quartics, 72 quintics, 336 binomials of degree 6,48 of degree 7, and 18 of degree 8. Out of this set, the applicable Markov moves are the same as in the case $\rho_{i j}=0$ with a few degree-six binomials added, such as: $p_{12}(0,0) p_{13}(1,1) p_{14}(1,1) p_{23}(0,1) p_{24}(1,0) p_{34}(0,0)-$ $p_{12}(1,1) p_{13}(0,1) p_{14}(1,0) p_{23}(0,0) p_{24}(0,0) p_{34}(1,1)$.

3.2.3. Case III: edge-dependent reciprocation. $\left(\rho_{i j}=\rho+\rho_{i}+\rho_{j}\right)$

To construct the design matrix $\mathcal{E}_{n}$ for this case, we start with the matrix $\mathcal{C}_{n}$ from the case $\rho_{i j}=\rho$, and introduce $n$ more rows indexed by $\rho_{1}, \ldots, \rho_{n}$. Every fourth column of the new matrix, indexed by $p_{i j}(1,1)$, has two nonzero entries: a 1 in the rows corresponding to $\rho_{i}$ and $\rho_{j}$. For example, when $n=2$, the matrix looks like this:

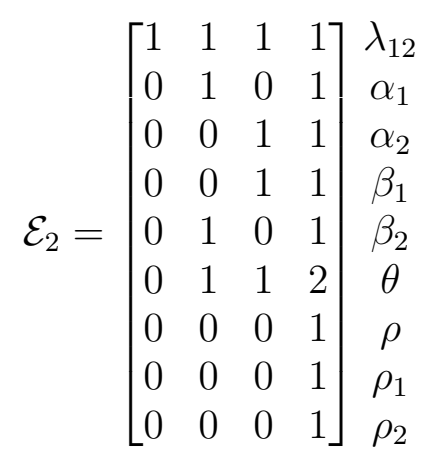

This is a full-rank matrix so the ideal for the 2-node network is empty.

With $n=3$ we get the expected result; the ideal $I_{\mathcal{E}_{3}}$ is the principal ideal

$$
I_{\mathcal{E}_{3}}=\left(p_{12}(1,0) p_{23}(1,0) p_{13}(0,1)-p_{12}(0,1) p_{23}(0,1) p_{13}(1,0)\right) .
$$

With $n=4$ we get the first interesting Markov moves for the edge-dependent case. The software 4 ti2 outputs a minimal generating set of the ideal $I_{\mathcal{E}_{4}}$ consisting of: 4 cubics, 18 quartics, and 24 quintics. The cubics, as usual, represent re-orienting a 3-cycle. Similarly some of the quartics represent 4-cycles. And then we get a few more binomials, of the following types:

$p_{13}(0,0) p_{24}(0,0) p_{14}(0,1) p_{23}(0,1)-p_{13}(0,1) p_{24}(0,1) p_{14}(0,0) p_{23}(0,0)$ of degree four, and $p_{13}(0,0) p_{24}(0,0) p_{14}(0,1) p_{12}(1,0) p_{23}(1,0)-p_{13}(1,0) p_{24}(0,1) p_{14}(0,0) p_{12}(0,1) p_{23}(0,0)$ of degree five. Note that these two are just representatives; we may, for example, replace every $p_{i j}(0,0)$ in each of them by $p_{i j}(1,1)$, and get other Markov moves which are minimal generators of the toric ideal $I_{\mathcal{E}_{4}}$.

3.3. From the $p_{1}$ model to an edge subring of a graph. A careful reader will have noticed a pattern in the moves that have appeared so far. To that end, let us single out two special submatrices that appear in the design matrices in each of the three cases.

1) First, for each case, we consider the matrix of the simplified model obtained from the $p_{1}$ model by simply forgetting the normalizing constants $\lambda_{i j}$. Let us denote these simplified 
matrices by $\tilde{\mathcal{Z}}_{n}, \tilde{\mathcal{C}}_{n}$ and $\tilde{\mathcal{E}}_{n}$. Note that ignoring $\lambda_{i j}$ 's results in zero columns for each column indexed by $p_{i j}(0,0)$, and so we are effectively also ignoring all $p_{i j}(0,0)$ 's. Hence, the matrices $\tilde{\mathcal{Z}}_{n}, \tilde{\mathcal{C}}_{n}$ and $\tilde{\mathcal{E}}_{n}$ have $\left(\begin{array}{l}n \\ 2\end{array}\right)$ less rows and $\left(\begin{array}{l}n \\ 2\end{array}\right)$ less columns than $\mathcal{Z}_{n}, \mathcal{C}_{n}$ and $\mathcal{E}_{n}$, respectively.

2) The second special matrix will be denoted by $\mathcal{A}_{n}$ and is common to all three cases. It is obtained from $\tilde{\mathcal{Z}}_{n}, \tilde{\mathcal{C}}_{n}$ or $\tilde{\mathcal{E}}_{n}$ by ignoring the columns indexed by $p_{i j}(1,1)$ for all $i$ and $j$, and then removing any zero rows.

While these constructions may seem artificial at a first glance, we will soon see that they are helpful in effectively describing Markov bases for $p_{1}$ model for any $n$.

Let us consider an example. The ideal of $I_{\mathcal{A}_{4}}$ is generated by the four cubics representing the 3-cycles, and six quadrics, each of which represents the following move for some choice of $i, j, k, l \in\{1, \ldots, 4\}$ :

Replace the edges $(i, j)$ and $(l, k)$ by the edges $(i, k)$ and $(l, j)$.

Graphically, the move simply exchanges the heads of the directed arrows while keeping the tails fixed, as illustrated in Figure 3.2 .

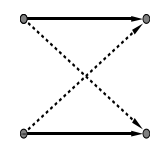

Figure 3.2. A degree-two move: dashed edges replaced by full edges.

It turns out that these moves are basic building blocks for the Markov moves for the 4node network, decorated with some other edges to ensure homogeneity, and sometimes this decoration can be quite non-obvious. They depend on the homogeneity requirements which are there for all three cases of $p_{1}$, but also on the way that bi-directed edges might interact with "regular" edges, specially in the case of no reciprocation. In particular, we will see (Theorems 3.11, 3.15) that the ideals of the simplified models are a sum of the ideal $I_{\mathcal{A}_{n}}$ and another nice toric ideal. It will then follow that the ideal of our model is a multi-homogeneous piece of the ideal of the simplified model (Theorem 3.21). Equivalently, the corresponding varieties are obtained by slicing the simplified-model varieties with hyperplanes. The upshot of the decomposition is that the Markov moves can be obtained by overlapping simple moves.

Example 3.2. The following binomial is a generator of the ideal $I_{\mathcal{Z}_{4}}$ :

$$
p_{12}(1,0) p_{13}(1,1) p_{23}(1,0) p_{24}(1,0)-p_{12}(0,1) p_{13}(1,0) p_{14}(1,0) p_{23}(1,1) \text {. }
$$

The move itself, is equivalent to performing a sequence of two simple moves, as illustrated in Figure 3.3 ,

replace the cycle $(1,2,3,1)$ by the cycle $(1,3,2,1)$,

followed by

replace the edges $(1,3)$ and $(2,4)$ by the edges $(1,4)$ and $(2,3)$.

This "decomposition" depends on the fact that reciprocation is zero, so that the double edge is valued the same as two regular edges. 

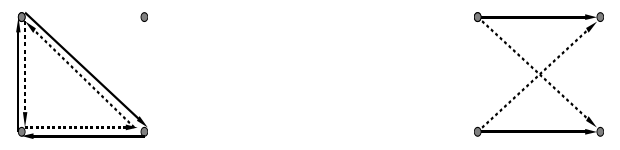

Figure 3.3. A sequence of two moves on 4 nodes: dashed edges are replaced by full edges.

The following example illustrates that not all Markov moves are obtained in the same fashion.

Example 3.3. Consider the case of edge-dependent reciprocation on $n=4$ nodes. The following degree-five binomial appears as a minimal generator of the ideal $I_{\mathcal{E}_{4}}$, for a choice of $1 \leq i, j, k, l \leq n$ :

$$
p_{i j}(1,0) p_{i k}(0,0) p_{i l}(0,1) p_{j k}(1,0) p_{j l}(0,0)-p_{i j}(0,1) p_{i k}(1,0) p_{i l}(0,0) p_{j k}(0,0) p_{j l}(0,1) .
$$

Clearly this move can be obtained by the following sequence of simple moves, illustrated in Figure 3.4:

Replace the edges $(1, i)$ and $(j, k)$ by the edges $(l, k)$ and $(j, i)$,

followed by

replace the edges $(i, j)$ and $(l, k)$ by the edges $(i, k)$ and $(l, j)$.
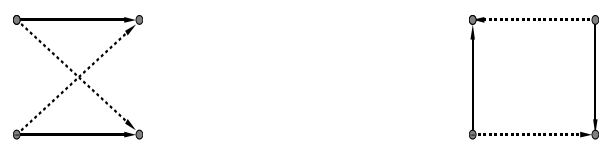

Figure 3.4. A sequence of two moves on 4 nodes: dashed edges are replaced by full edges.

Note that we do not remove or add the empty edge represented by $p_{i j}(0,0)$; but these variables are required by homogeneity.

\subsection{The toric ideal of the common submatrix. $\left(\mathcal{A}_{n}\right)$}

Focusing on the submatrix $\mathcal{A}_{n}$ reveals additional structure which can be studied using some standard algebraic techniques. To that end, we recall the following standard definition (see [20]).

Definition 3.4. Let $k$ be any field (e.g. $k=\mathbb{C}$ ), $G$ be any graph and $E(G)$ the set of its edges. If we consider the vertices of $G$ to be variables, then the edges of $G$ correspond to degree-two monomials in those variables. The ring

$$
k[G]:=k[x y \mid(x, y) \in E(G)]
$$

is called the monomial subring or the edge subring of the graph $G$. Its ideal of relations is called the toric ideal of the edge subring. 
We will be interested in special graphs. Let $G_{n}:=K_{n, n} \backslash\{(i, i)\}_{i=1}^{n}$ be the complete bipartite (undirected) graph $K_{n, n}$ on two sets of with $n$ vertices each, but with the vertical edges $(i, i)$ removed. If we label one set of vertices $\alpha_{1}, \ldots, \alpha_{n}$ and the other set $\beta_{1}, \ldots, \beta_{n}$, then our graph $G_{n}$ has edges $\left(\alpha_{i}, \beta_{j}\right)$ for all $i \neq j$. Thus we are interested in the ring $k\left[G_{n}\right]:=k\left[\alpha_{i} \beta_{j} \mid\left(\alpha_{i}, \beta_{j}\right)\right.$ is an edge of $\left.G_{n}\right]$.

Lemma 3.5. Adopting the notation above, $I_{\mathcal{A}_{n}}$ is the toric ideal of $k\left[G_{n}\right]$.

Proof. Returning to our definition of $\mathcal{A}_{n}$, we see that it is the incidence matrix of the graph $G_{n}$; that is, the columns of the matrix correspond to the exponents of the monomials representing the edges of $G_{n}$. Thus the claim readily follows by the definition of the toric ideal of $k\left[G_{n}\right]$ from Section 8.1. in [20].

We will use this correspondance to obtain a Gröbner basis of $I_{\mathcal{Z}_{n}}$ and $I_{\mathcal{E}_{n}}$. But first, let us introduce one more concept which is crucial to the description of a Gröbner basis of $I_{\mathcal{A}_{n}}$.

Definition 3.6. Following [5] and [20], we say that a binomial $f_{c}$ arises from a cycle $c$ of $G_{n}$ if:

1) $c$ is a cycle of the graph $G_{n}$; namely, $c$ is a closed walk on a subset of the vertices of $G_{n}$ such hat no vertex is visited twice: $c=\left(\alpha_{i_{1}}, \beta_{j_{1}}, \alpha_{i_{2}}, \beta_{j_{2}}, \ldots, \beta_{j_{k}}, \alpha_{i_{1}}\right)$,

2) $f_{c}$ is the binomial obtained by placing the variables corresponding to the edges of the cycle $c$ alternately in one and then the other monomial of $f_{c}$; that is, $f_{c}:=f_{c}^{+}-f_{c}^{-}$with $f_{c}^{+}:=$ $p_{i_{1} j_{1}}(1,0) p_{i_{2} j_{2}}(1,0) \ldots p_{i_{k-1} j_{k-1}}(1,0) p_{i_{i} j_{k}}(1,0)$ and $f_{c}^{-}:=p_{i_{2} j_{1}}(1,0) p_{i_{3} j_{2}}(1,0) \ldots p_{i_{k} j_{k-1}}(1,0) p_{i_{1} j_{k}}(1,0)$, where for ease of notation we have let $p_{i j}(1,0)=p_{j i}(0,1)$ if $i>j$.

There are finitely many cycles in $G_{n}$, though they may be nontrivial to enumerate. However, we will use this description to provide (theoretically) an explicit Gröbner basis for our toric ideal. In practice, one can use the program 4ti2 to obtain the binomials fairly quickly. Gröbner bases, and even Graver bases, for the ideals $I_{\mathcal{A}_{n}}$ are known ([5], [20]):

Theorem 3.7 (Bases of the ideal of the common submatrix). Let $\mathcal{G}_{n}$ be the set of binomials arising from the cycles of the graph $G_{n}$. Then $\mathcal{G}_{n}$ is a Gröbner basis of $I_{\mathcal{A}_{n}}$. Thus it is also a Markov basis of $I_{\mathcal{A}_{n}}$, but not necessarily a minimal one. Moreover, this set is a Graver basis of the ideal, and it coincides with the universal Gröbner basis.

Proof (outline). We will use Lemma 3.5 and the appropriate results from [5] and [20] about the toric ideals of bipartite graphs. Note that there is quite a bit of vocabulary used in the cited results, but we have only defined those terms which we use in our description of the Gröbner basis in the Theorem.

Oshugi and Hibi in [5] (Lemma 3.1.), and also Villarreal in [20] (Proposition 8.1.2) give a generating set for $I_{\mathcal{A}_{n}}$. Moreover, Lemma 1.1. of [5] implies that these generators are in fact the Graver basis for $I_{\mathcal{A}_{n}}$. On the other hand, from Chapter 8 of [20] we see that precisely these generators in fact correspond to circuits, as well as the universal Gröbner basis for $I_{\mathcal{A}_{n}}$. It follows that the circuits equal the Graver basis for our ideal $I_{\mathcal{A}_{n}}$. Avoiding technical details, we will just state that circuits are a special subset of the Graver basis: they are minimal with respect to inclusion. The binomials in the Graver basis are given by the even cycles of the graph $G_{n}$ (Corollary 8.1.5. in [20]). But since our graph is bipartite, all of the cycles are even (Proposition 6.1.1. in [20]), so it suffices to say that the Graver basis of $I_{\mathcal{A}_{n}}$ 
consists of binomials arising from the cycles of the graph $G_{n}$. Since the universal Gröbner basis (which equals Graver basis in this case) is by definition a Gröbner basis with respect to any term order, the proof is complete. The binomials arising from the cycles of the graph $G_{n}$ form the desired set $\mathcal{G}_{n}$.

In addition, we have a nice recursive way of constructing the binomials in $\mathcal{G}_{n}$.

Proposition 3.8. The set $\mathcal{G}_{n}$ consists of precisely the binomials in $\mathcal{G}_{n-1}$ for all of the $n-1$ node subnetworks, together with the binomials arising from the cycles of the graph $G_{n}$ which pass through either $\alpha_{i}$ or $\beta_{i}$ for each $i$ between 1 and $n$.

Proof. The last condition can be restated as follows: we are looking for the primitive binomials $f$ such that for each node $i$ in the random network, there exists an edge $(i, j)$ such that the variable $p_{i j}(a, b)$ appears in one of the monomials of $f$. The reduction to the $n-1$-node subnetworks is immediate from Proposition 4.13. in [19]. Namely, the design matrices for the $n$-1-node networks are submatrices of $\mathcal{A}_{n}$, hence the Graver basis of $I_{\mathcal{A}_{n-1}}$ equals that of $I_{\mathcal{A}_{n}}$ involving at most $n-1$ nodes.

An example of a degree 5 binomial in $I_{\mathcal{A}_{5}}$ which uses all 5 nodes is

$$
p_{14}(1,0) p_{15}(0,1) p_{23}(1,0) p_{24}(0,1) p_{35}(1,0)-p_{14}(0,1) p_{15}(1,0) p_{23}(0,1) p_{24}(1,0) p_{35}(0,1) .
$$

The first term represents a cycle $(1,4,2,3,5)$, while the second term represents its inverse, $(1,5,3,2,4)$. In terms of the graph $G_{5}$, each of the monomials represent edges, in alternating order, of the cycle $\left(\alpha_{1}, \beta_{4}, \alpha_{2}, \beta_{3}, \alpha_{5}, \beta_{1}, \alpha_{4}, \beta_{2}, \alpha_{3}, \beta_{5}, \alpha_{1}\right)$.

Remark 3.9. In the Proposition above, all binomials have squarefree terms. This means that the initial ideal of the toric ideal is squarefree. Using a theorem of Hochster ([14]), this implies that the coordinate rings of the corresponding varieties are arithmetically CohenMacaulay. This is a powerful algebraic-geometric property that is not encountered too often.

Remark 3.10. There is a special property of the design matrix, called unimodularity which, if satisfied, implies that all initial ideals or its toric ideal are squarefree, and also that circuits equal the Graver basis. In general, for the design matrix of the $p_{1}$ model it does not hold.

We are now ready to embark on a more detailed study of the simplified models.

\subsubsection{Case I: no reciprocation. $\left(\rho_{i j}=0\right.$, simplified model)}

Let's start with the simplest nontrivial example: $n=2$. The rows of the design matrix $\tilde{\mathcal{Z}}_{2}$ are $[1,0,1],[0,1,1],[0,1,1],[1,0,1],[1,1,2]$, and are indexed by $\alpha_{1}, \alpha_{2}, \beta_{1}, \beta_{2}$, and $\theta$, while the columns are indexed by $p_{12}(1,0), p_{12}(0,1), p_{12}(1,1)$. One easily checks that the ideal $I_{\tilde{\mathcal{Z}}_{2}}$ is the principal ideal $I_{\tilde{\mathcal{Z}}_{2}}=\left(p_{12}(1,0) p_{12}(0,1)-p_{12}(1,1)\right)$ and thus this single binomial is a Markov basis, and also a Gröbner basis with respect to any term order.

Next, let $n=3$. The toric ideal $I_{\tilde{\mathcal{Z}}_{3}}$ is minimally generated by the following set of binomials: $p_{23}(0,1) p_{23}(1,0)-p_{23}(1,1), p_{13}(0,1) p_{13}(1,0)-p_{13}(1,1), p_{12}(0,1) p_{12}(1,0)-p_{12}(1,1)$, $p_{12}(0,1) p_{13}(1,0) p_{23}(0,1)-p_{12}(1,0) p_{13}(0,1) p_{23}(1,0)$. It is interesting to note that the first 3 generators are the "trivial" ones (as seen in case $n=2$ ). 
For the network on $n=4$ nodes, we get the first interesting Markov basis elements. Namely, there are inhomogeneous binomials of degree 2 which represent the "obvious" relations, which are of the form $p_{i j}(0,1) p_{i j}(1,0)-p_{i j}(1,1)$. Next, there are squarefree quadrics (homogeneous binomials of degree 2): $p_{i k}(1,0) p_{j l}(1,0)-p_{i l}(1,0) p_{j k}(1,0)$, and there are degree-three binomials that resemble the degree-three generator of the ideal $I_{\tilde{\mathcal{Z}}_{3}}$ :

$p_{i j}(0,1) p_{i k}(1,0) p_{j k}(0,1)-p_{i j}(1,0) p_{i k}(0,1) p_{j k}(1,0)$. Note that if we write $p_{j k}(1,0)$ and $j>k$, then we mean $p_{k j}(0,1)$, since for all $p_{j k}(a, b)$ we assume $j<k$.

In order to obtain a Gröbner basis for the ideal $I_{\tilde{\mathcal{Z}}_{n}}$, we can use what we know about $I_{\mathcal{A}_{n}}$. The advantage of studying $I_{\mathcal{A}_{n}}$ over $I_{\tilde{\mathcal{Z}}_{n}}$ is that it is a homogeneous ideal; that is, both monomials in each binomial appear with the same degree. One can see that the ideal is homogeneous by inspecting the columns of the matrix: each column has the same 1-norm. The ideal of the simplified model admits a nice decomposition:

Theorem 3.11 (Decomposition of the ideal of the simplified model $\tilde{\mathcal{Z}}_{n}$ ). With the notation as above, $I_{\tilde{\mathcal{Z}}_{n}}=I_{\mathcal{A}_{n}}+T$, where $T$ is the ideal generated by the binomials of the form $p_{i j}(0,1) p_{i j}(1,0)-p_{i j}(1,1)$ for all pairs of nodes $i<j$.

Proof. One inclusion $(\supset)$ is clear. To prove the other inclusion, consider $f \in I_{\tilde{\mathcal{Z}}_{n}}$ such that $p_{i j}(1,1)$ divides one of its terms for some $i, j$. Since the ideal is toric, it suffices to consider the case when $f$ is a binomial. Then it can be written as $f=p_{i j}(1,1) m_{1}-m_{2}$ where $m_{1}$ and $m_{2}$ are monomials. But then we can write $f=\left(p_{i j}(1,0) p_{i j}(0,1) m_{1}-m_{2}\right)-$ $m_{1}\left(p_{i j}(0,1) p_{i j}(1,0)-p_{i j}(1,1)\right)$. Repeating this procedure if necessary, we can write $f$ as a combination of binomials whose terms are not divisible by any of the variables $p_{i j}(1,1)$ and those binomials that generate $T$. To conclude, note that those not divisible by any of the $p_{i j}(1,1)$ are in the ideal $I_{\mathcal{A}_{n}}$ by definition.

Combining the above results, we obtain a Markov (Gröbner) basis for the ideal of the simplified model:

Theorem 3.12 (Gröbner basis for the simplified model $\tilde{\mathcal{Z}}_{n}$ ). Let $\mathcal{G}_{T}$ be the binomial generators of $T$, that $i s, \mathcal{G}_{T}:=\left\{p_{i j}(0,1) p_{i j}(1,0)-p_{i j}(1,1)\right\}$ for all pairs of nodes $i<j$. Let $\mathcal{G}_{n}$ be the set of binomials arising from the cycles of $G_{n}$, as in Theorem (3.7). Then the ideal of the simplified model $I_{\tilde{\mathcal{Z}}_{n}}$ has a Gröbner basis, and thus a Markov basis, consisting of the union of the binomials in $\mathcal{G}_{n}$ and $\mathcal{G}_{T}$.

Proof. First we will show that the set $\mathcal{G}_{T}$ actually forms a Gröbner basis for $T$. Namely, pick an elimination order where the variables $p_{i j}(1,1)$ are greater then the remaining variables. Then, the degree-one terms of the binomials in $\mathcal{G}_{T}$ are the initial terms. Since they are all relatively prime, the given generators form a Gröbner basis as all S-pairs reduce to zero (for details about Gröbner basis computations, the reader should refer to Buchberger's criterion [3] as well as standard references [6] and [19]).

Next, take any Gröbner basis $\mathcal{G}_{n}$ for the ideal $I_{\mathcal{A}_{n}}$ with respect to some order $\prec$. According to Lemma 3.11, $\mathcal{G}_{n} \cup \mathcal{G}_{T}$ generates the ideal $I_{\tilde{\mathcal{Z}}_{n}}$. Let $\prec^{\prime}$ be the refinement of the term order $\prec$ by the elimination order used for $\mathcal{G}_{T}$. The we get that $\mathcal{G}_{n} \cup \mathcal{G}_{T}$ is in fact a Gröbner basis for $I_{\tilde{\mathcal{Z}}_{n}}$ with respect to $\prec^{\prime}$, since no initial term in $\mathcal{G}_{T}$ appears as a term in $\mathcal{G}_{n}$ and thus all the S-pairs will again reduce to zero. The Gröbner basis $\mathcal{G}_{n}$ for the ideal $I_{\mathcal{A}_{n}}$ follows from Theorem 3.7). 
To derive minimal Markov bases for the simplified model, it remains to find a minimal generating set for the ideal of the edge subring of an incomplete bipartite graph. To the best of our knowledge, there isn't a result that states these are generated in degrees two and three. In particular, we claim:

Conjecture 3.13. A minimal Markov basis for the ideal $I_{\tilde{\mathcal{Z}}_{n}}$ consists of the elements of degrees 2 and 3. All inhomogeneous elements are of the form $p_{i j}(0,1) p_{i j}(1,0)-p_{i j}(1,1)$. All quadrics are of the form $p_{i k}(1,0) p_{j l}(1,0)-p_{i l}(1,0) p_{j k}(1,0)$, where $i, j, k, l$ vary over all possible four-node subnetworks. All cubics are of the form $p_{i j}(0,1) p_{i k}(1,0) p_{j k}(0,1)-$ $p_{i j}(1,0) p_{i k}(0,1) p_{j k}(1,0)$, where $i, j$, and $k$ vary over all possible triangles (3-cycles) in the network.

\subsubsection{Case II: constant reciprocation. $\left(\rho_{i j}=\rho\right.$, simplified model)}

There is a small but crucial difference between $\tilde{\mathcal{Z}}_{n}$ and $\tilde{\mathcal{C}}_{n}$ : one more row, representing the constant $\rho$ added to those columns indexed $p_{i j}(1,1)$ for all $i, j \in[n]$. This row is filled with the pattern 0,0,1 repeated as many times as necessary. Note that adding the extra row makes this ideal homogeneous, that is, the two terms of each binomial have the same degree. (Note that homogeneity is easy to verify: the dot product of each column of $\tilde{\mathcal{C}}_{n}$ with the vector $w=[1, \ldots, 1,-n-1]$ results in the vector $[1, \ldots, 1]$, as required, for example, by Lemma 4.14. in [19].) For two nodes, the ideal is trivial. For $n=3$ nodes, the Markov basis consists of 4 binomials of degree 3 of the following forms: $p_{i k}(1,0) p_{i k}(0,1) p_{j k}(1,1)-$ $p_{i k}(1,1) p_{j k}(1,0) p_{j k}(0,1), p_{i j}(1,0) p_{i k}(0,1) p_{j k}(1,0)-p_{i j}(0,1) p_{i k}(1,0) p_{j k}(0,1)$, and 6 of degree 4 of the form: $p_{i j}(0,1)^{2} p_{i k}(1,1) p_{j k}(0,1)-p_{i j}(1,1) p_{i k}(0,1)^{2} p_{j k}(1,0)$. For $n \geq 4$, the Markov bases consist of elements of the above type, but also include quadrics of the form: $p_{i k}(1,0) p_{j l}(1,0)-p_{i l}(1,0) p_{j k}(1,0), p_{i j}(1,1) p_{k l}(1,1)-p_{i k}(1,1) p_{j l}(1,1)$.

Conjecture 3.14 (Minimal Markov basis of the simplified model $\tilde{\mathcal{C}}_{n}$ ). The ideal $I_{\tilde{\mathcal{C}}_{n}}$ is generated in degrees 2, 3, and 4, and the binomials are of the form described above.

Note: due to the existence of $\rho_{i j}$, we do not get the relations $\mathcal{G}_{T}$ from the case $\rho_{i j}=0$. Recall they were of the form $p_{i j}(1,0) p_{i j}(0,1)-p_{i j}(1,1)$. They cannot be in the ideal in this case, since the bi-directed edge between $i$ and $j$ is valued differently then the two single edges $(i, j)$ and $(j, i)$. Gröbner bases or even Markov bases in this case remain an open problem that we will continue to study.

\subsubsection{Case III: edge-dependent reciprocation. $\left(\rho_{i j}=\rho+\rho_{i}+\rho_{j}\right.$, simplified model $)$}

To construct the design matrices $\tilde{\mathcal{Z}}_{n}$ for this case, we start with the matrix $\tilde{\mathcal{C}}_{n}$ from the case $\rho_{i j}=\rho$, and introduce $n$ more rows indexed by $\rho_{1}, \ldots, \rho_{n}$. Every third column of the new matrix, indexed by $p_{i j}(1,1)$, has two nonzero entries: a 1 in the rows corresponding to 
$\rho_{i}$ and $\rho_{j}$. For example, when $n=3$, the matrix is:

$$
\tilde{\mathcal{E}}_{3}=\left[\begin{array}{lllllllll}
1 & 0 & 1 & 1 & 0 & 1 & 0 & 0 & 0 \\
0 & 1 & 1 & 0 & 0 & 0 & 1 & 0 & 1 \\
0 & 0 & 0 & 0 & 1 & 1 & 0 & 1 & 1 \\
0 & 1 & 1 & 0 & 1 & 1 & 0 & 0 & 0 \\
1 & 0 & 1 & 0 & 0 & 0 & 0 & 1 & 1 \\
0 & 0 & 0 & 1 & 0 & 1 & 1 & 0 & 1 \\
1 & 1 & 2 & 1 & 1 & 2 & 1 & 1 & 2 \\
0 & 0 & 1 & 0 & 0 & 1 & 0 & 0 & 1 \\
0 & 0 & 1 & 0 & 0 & 1 & 0 & 0 & 0 \\
0 & 0 & 1 & 0 & 0 & 0 & 0 & 0 & 1 \\
0 & 0 & 0 & 0 & 0 & 1 & 0 & 0 & 1
\end{array}\right] .
$$

The kernel of this matrix is generated by one vector, and in fact the ideal $I_{\tilde{\mathcal{E}}_{3}}$ is the principal ideal $I_{\tilde{\mathcal{E}}_{3}}=\left(p_{12}(1,0) p_{23}(1,0) p_{13}(0,1)-p_{12}(0,1) p_{23}(0,1) p_{13}(1,0)\right)$. When we calculate the ideals for larger networks, some more familiar binomials appear.

Theorem 3.15 (Decomposition of the simplified model $\tilde{\mathcal{E}}_{n}$ ). Let $I_{\mathcal{A}_{n}}$ be the ideal as in Section 3.4. Let $Q$ be the ideal generated by the quadrics $p_{i j}(1,1) p_{k l}(1,1)-p_{i k}(1,1) p_{j l}(1,1)$ for each set of indices $1 \leq i, j, k, l \leq n$. Then $I_{\tilde{\mathcal{E}}_{n}}=I_{\mathcal{A}_{n}}+Q$ for every $n \geq 4$.

Proof. Consider the submatrix of $\tilde{\mathcal{E}}_{n}$ consisting of those columns that have only zeros in the last $n$ rows. Erasing those zeros, we get precisely the columns of $\mathcal{A}_{n}$. Thus $I_{\tilde{\mathcal{E}}_{n}} \supset I_{\mathcal{A}_{n}}$.

Similarly, the ideal of relations among those columns that have nonzero entries in the last $n$ rows is also contained in $I_{\tilde{\mathcal{E}}_{n}}$. We will now show that this ideal equals $Q$. To simplify notation, let $M$ be the matrix consisting of the columns in question, that is, those that are indexed by $p_{i j}(1,1)$ for all pairs $i<j$. Recalling the definition of the action of the simplified parametrization map on $p_{i j}(1,1): p_{i j}(1,1) \mapsto \alpha_{i} \beta_{j} \alpha_{j} \beta_{i} \theta \rho \rho_{j} \rho_{j}$, and the fact that the last $n$ rows of $M$ are indexed by $\rho_{1}, \ldots, \rho_{n}$, we see that to study the toric ideal $I_{M}$ it suffices to study the ideal $I_{M^{\prime}}$ where $M^{\prime}$ is the submatrix consisting of the last $n$ rows of $M$. But $I_{M^{\prime}}$ is a well-studied toric ideal! Namely, $M^{\prime}$ agrees with the incidence matrix of the complete graph $K_{n}$ on $n$ vertices: for each pair $\{i, j\}$ there exists an edge $(i, j)$. Therefore the toric ideal $I_{M^{\prime}}$ agrees with the toric ideal of the edge ring $k\left[K_{n}\right]$, where the vertices of $K_{n}$ are labelled by the nodes $1, \ldots, n$, and an edge between $i$ and $j$ represents the variable $p_{i j}(1,1)$. It is well-known (see, for example, [5]) that $I_{M^{\prime}}$ is generated by quadrics. Moreover, from [4] and references given therein (see also Corollary 9.2.3. in [20]) it follows that these quadrics be interpreted as $2 \times 2$-minors of a certain tableaux. But our definition of the ideal $Q$ is precisely the set of those $2 \times 2$-minors, thus $I_{M^{\prime}}=Q$ and we obtain $I_{\tilde{\mathcal{E}}_{n}} \supset Q$.

To complete the proof, we need to show the reverse inclusion. To that end, let $f=$ $f^{+}-f^{-} \in I_{\tilde{\mathcal{E}}_{n}}$. If no $p_{i j}(1,1)$ divides either term of $f$, then $f \in I_{\mathcal{A}_{n}}$ and we are done. On the other hand, suppose $p_{i j}(1,1) \mid f^{+}$for some pair $i, j$. Then the definition of $I_{\tilde{\mathcal{E}}_{n}}$ implies that one of the following conditions are satisfied:

1) $p_{i j}(1,1) \mid f^{-}$. But then the binomial $f$ is not primitive, and thus it cannot be required in any minimal generating set, or a Gröbner basis, of $I_{\tilde{\mathcal{E}}_{n}}$.

2) $p_{k l}(1,1) \mid f^{+}$for some other pair of indices $k, l$. But this in turn implies that 
i) $p_{i j}(1,1) p_{k l}(1,1) \mid f^{-}$and $f$ fails to be primitive trivially; or

ii) without loss of generality, $p_{i k}(1,1) p_{j l}(1,1) \mid f^{-}$and $f$ fails to be primitive by the quadric in $Q$; or

iii) $f^{+}$is divisible by another variable $p_{s t}(1,1)$, and the pattern continues.

In general, it is clear that any $f$ whose terms are divisible by the variables representing columns of $M$ will fail to be primitive by one of the binomials in the ideal $Q$. Therefore, the ideal $I_{\tilde{\mathcal{E}}_{n}}$ is generated by the binomials of $I_{\mathcal{A}_{n}}$ and $Q$, as desired.

Next we obtain a Gröbner basis for our toric ideal. Recall from Section 3.4 that $G_{n}=$ $K_{n, n} \backslash\{(i, i)\}_{i=1}^{n}$. We have seen that the graph $G_{n}$ played an essential role in the case when $\rho_{i j}=0$, and it will play an essential role here as well, since it is essential in describing the Gröbner bases of the common submatrix $\mathcal{A}_{n}$. However, in order to study the Gröbner basis of the ideal $Q$, we need to use graphs with more edges. It comes as no surprise, then, that these ideals will have a more complicated Gröbner basis then those from Section 3.4. Thus we need to generalize Definition 3.6 for the complete graph $K_{n}$ on $n$ vertices:

Definition 3.16. As before, following [5] and [20], we say that a binomial $f_{w}$ arises from an even closed walk $w$ of $K_{n}$ if:

1) $w$ is an even closed walk $w$ of $K_{n}$; namely, $w$ is a closed walk on a subset of the vertices of $K_{n}$ such that it is of even length (even number of edges) $w=\left(\left\{v_{1}, v_{2}\right\}, \ldots\left\{v_{2 k-1}, v_{2 k}\right\}\right.$ ), with $1 \leq v_{1}, \ldots, v_{2 k} \leq n$. The closed condition requires that $v_{2 k}=v_{1}$.

2) $f_{w}$ is the binomial obtained by placing the variables corresponding to the edges of the walk $w$ alternately in one and then the other monomial of $f_{w}$. In other words, $f_{w}:=f_{w}^{+}-f_{w}^{-}$with $f_{w}^{+}:=p_{v_{1}, v_{2}}(1,1) p_{v_{3}, v_{4}}(1,1) \ldots p_{v_{2 k-1}, v_{1}}(1,1)$ and $f_{w}^{-}:=p_{v_{2}, v_{3}}(1,1) p_{v_{4}, v_{5}}(1,1) \ldots p_{v_{2 k-2}, v_{2 k-1}}(1,1)$, where for compactness of notation we have let $p_{i j}(1,1)=p_{j i}(1,1)$ if $i>j$.

We say that the even closed walk $w$ is primitive if the corresponding binomial is primitive.

Let us state the characterization of such walks from [5]:

Lemma 3.17 ([5], Lemma 3.2.). A primitive even closed walk on a graph $G$ is one of the following: an even cycle of $G$; or two odd cycles having exactly one common vertex; or two odd cycles having no common vertex together with two more walks, both of which connect a vertex of the first cycle with a vertex of the second cycle.

In Section 3.4, we have seen only even cycles. This is because the graph in question, namely $G_{n}$, was bipartite, and therefore had no odd cycles. We are now ready to state the main result of this section.

Theorem 3.18 (Gröbner basis of the simplified model $\tilde{\mathcal{E}}_{n}$ ). The ideal $I_{\tilde{\mathcal{E}}_{n}}$ admits a Gröbner basis consisting of the binomials arising from the cycles of the bipartite graph $G_{n}$ together with the binomials arising from the primitive closed even walks of $K_{n}$, the complete graph on $n$ vertices.

Proof. Recall that $\mathcal{G}_{n}$ are precisely the binomials arising from the cycles of $G_{n}$. We have seen in Theorem 3.12 that $\mathcal{G}_{n}$ form a Gröbner basis for $I_{\mathcal{A}_{n}}$ (in fact, they are a Graver basis). Since the ideals $I_{\mathcal{A}_{n}}$ and $Q$ are in disjoint sets of variables, it remains to find a Gröbner basis for $Q$. We do this by generalizing the argument in the proof of Theorem 3.12 . Namely, from the proof of Theorem 3.15 we know that $Q$ is the toric ideal of the edge ring $k\left[K_{n}\right]$. This 
allows us to use [5] and [20] again, and we obtain (e.g. Lemmas 3.1. and 3.2. in [5]) that the Graver basis of $Q$ consists of the binomials arising from the primitive even closed walks on $K_{n}$. In addition, note that Theorem 9.2.1 of [20] provides a quadratic Gröbner basis as well.

A proof of Conjecture 3.13 would imply the following:

Conjecture 3.19 (Minimal Markov basis of the simplified model $\tilde{\mathcal{E}}_{n}$ ). For $n \geq 4$, the ideal $I_{\tilde{\mathcal{E}}_{n}}$ is minimally generated by homogeneous binomials of degrees 2 and 3 . More precisely, the degree 2 and 3 binomials in $\mathcal{G}_{n}$, together with the quadratic generators of $Q$, form a Markov basis for the model.

3.5. From the edge subring of a graph back to the $p_{1}$ model. We are now ready to introduce the $\lambda_{i j}$ back into the parametrization and consider the original design matrices $\mathcal{Z}_{n}, \mathcal{C}_{n}$ and $\mathcal{E}_{n}$. Recall that they can be obtained from $\tilde{\mathcal{Z}}_{n}, \tilde{\mathcal{C}}_{n}$ and $\tilde{\mathcal{E}}_{n}$, respectively, by adding $\left(\begin{array}{l}n \\ 2\end{array}\right)$ columns representing the variables $p_{i j}(0,0)$ for all $i<j$ and $\left(\begin{array}{l}n \\ 2\end{array}\right)$ rows indexed by $\lambda_{i j}$.

Example 3.20. Let $n=4$. Then, for the case in which $\rho_{i j}=0$, the $15 \times 24$ design matrix $\mathcal{Z}_{4}$ is:

$$
\left[\begin{array}{llllllllllllllllllllllll}
1 & 1 & 1 & 1 & 0 & 0 & 0 & 0 & 0 & 0 & 0 & 0 & 0 & 0 & 0 & 0 & 0 & 0 & 0 & 0 & 0 & 0 & 0 & 0 \\
0 & 0 & 0 & 0 & 1 & 1 & 1 & 1 & 0 & 0 & 0 & 0 & 0 & 0 & 0 & 0 & 0 & 0 & 0 & 0 & 0 & 0 & 0 & 0 \\
0 & 0 & 0 & 0 & 0 & 0 & 0 & 0 & 1 & 1 & 1 & 1 & 0 & 0 & 0 & 0 & 0 & 0 & 0 & 0 & 0 & 0 & 0 & 0 \\
0 & 0 & 0 & 0 & 0 & 0 & 0 & 0 & 0 & 0 & 0 & 0 & 1 & 1 & 1 & 1 & 0 & 0 & 0 & 0 & 0 & 0 & 0 & 0 \\
0 & 0 & 0 & 0 & 0 & 0 & 0 & 0 & 0 & 0 & 0 & 0 & 0 & 0 & 0 & 0 & 1 & 1 & 1 & 1 & 0 & 0 & 0 & 0 \\
0 & 0 & 0 & 0 & 0 & 0 & 0 & 0 & 0 & 0 & 0 & 0 & 0 & 0 & 0 & 0 & 0 & 0 & 0 & 0 & 1 & 1 & 1 & 1 \\
0 & 1 & 1 & 2 & 0 & 1 & 1 & 2 & 0 & 1 & 1 & 2 & 0 & 1 & 1 & 2 & 0 & 1 & 1 & 2 & 0 & 1 & 1 & 2 \\
0 & 1 & 0 & 1 & 0 & 1 & 0 & 1 & 0 & 1 & 0 & 1 & 0 & 0 & 0 & 0 & 0 & 0 & 0 & 0 & 0 & 0 & 0 & 0 \\
0 & 0 & 1 & 1 & 0 & 0 & 0 & 0 & 0 & 0 & 0 & 0 & 0 & 1 & 0 & 1 & 0 & 1 & 0 & 1 & 0 & 0 & 0 & 0 \\
0 & 0 & 0 & 0 & 0 & 0 & 1 & 1 & 0 & 0 & 0 & 0 & 0 & 0 & 1 & 1 & 0 & 0 & 0 & 0 & 0 & 1 & 0 & 1 \\
0 & 0 & 0 & 0 & 0 & 0 & 0 & 0 & 0 & 0 & 1 & 1 & 0 & 0 & 0 & 0 & 0 & 0 & 1 & 1 & 0 & 0 & 1 & 1 \\
0 & 0 & 1 & 1 & 0 & 0 & 1 & 1 & 0 & 0 & 1 & 1 & 0 & 0 & 0 & 0 & 0 & 0 & 0 & 0 & 0 & 0 & 0 & 0 \\
0 & 1 & 0 & 1 & 0 & 0 & 0 & 0 & 0 & 0 & 0 & 0 & 0 & 0 & 1 & 1 & 0 & 0 & 1 & 1 & 0 & 0 & 0 & 0 \\
0 & 0 & 0 & 0 & 0 & 1 & 0 & 1 & 0 & 0 & 0 & 0 & 0 & 1 & 0 & 1 & 0 & 0 & 0 & 0 & 0 & 0 & 1 & 1 \\
0 & 0 & 0 & 0 & 0 & 0 & 0 & 0 & 0 & 1 & 0 & 1 & 0 & 0 & 0 & 0 & 0 & 1 & 0 & 1 & 0 & 1 & 0 & 1
\end{array}\right]
$$

The following analysis applies to both the case of constant $\rho_{i j}$ and the case of edgedependent $\rho_{i j}$, thus we will treat them simultaneously.

There is a very concise way to describe the new toric ideal in terms of the cases when $\lambda_{i j}$ were ignored. For a binomial $f$, we will say that $f$ is multi-homogeneous with respect to each pair $\{i, j\}$ if the degrees in the variables indexed by the pair $\{i, j\}$ agree for the two monomials of $f$. More precisely, if $f=f^{+}-f^{-}$, then we require that $\operatorname{deg}_{p_{i j}(0,0)}\left(f^{+}\right)+\operatorname{deg}_{p_{i j}(1,0)}\left(f^{+}\right)+$ $\operatorname{deg}_{p_{i j}(0,1)}\left(f^{+}\right)+\operatorname{deg}_{p_{i j}(1,1)}\left(f^{+}\right)=\operatorname{deg}_{p_{i j}(0,0)}\left(f^{-}\right)+\operatorname{deg}_{p_{i j}(1,0)}\left(f^{-}\right)+\operatorname{deg}_{p_{i j}(0,1)}\left(f^{-}\right)+\operatorname{deg}_{p_{i j}(1,1)}\left(f^{-}\right)$ for each pair $\{i, j\}$. This allows us to make a simple observation.

Proposition 3.21 (Geometry of the $p_{1}$ model). The toric ideals $I_{\mathcal{Z}_{n}}, I_{\mathcal{C}_{n}}$ and $I_{\mathcal{E}_{n}}$ of the $p_{1}$ model on an $n$-node network are precisely those parts of the ideals $I_{\tilde{\mathcal{Z}}_{n}}, I_{\tilde{\mathcal{C}}_{n}}$ and $I_{\tilde{\mathcal{E}}_{n}}$, respectively, which are multi-homogeneous with respect to each pair $\{i, j\}$. Therefore, the toric 
variety for the $p_{1}$ models is obtained by slicing the corresponding varieties of the simplified model by $\left(\begin{array}{l}n \\ 2\end{array}\right)$ hyperplanes.

Proof. The rows indexed by $\lambda_{i j}$ that are added to the matrices $I_{\tilde{\mathcal{Z}}_{n}}, I_{\tilde{\mathcal{C}}_{n}}$ and $I_{\tilde{\mathcal{E}}_{n}}$ require precisely that the binomials in the ideal are multi-homogeneous according to the criterion given above. For example, consider the first row indexed by $\lambda_{1,2}$. For any binomial $f=$ $p^{u^{+}}-p^{u^{-}}$in the ideal, the exponent vector $u^{+}-u^{-}$being in the kernel of the matrix means that the number of variables $p_{1,2}(\bullet, \bullet)$ which appear in $p^{u^{+}}$equals the number of variables $p_{1,2}(\bullet, \bullet)$ that appear in $p^{u^{-}}$.

For the hyperplane section statement, note that the varieties defined by $I_{\tilde{\mathcal{Z}}_{n}}, I_{\tilde{\mathcal{C}}_{n}}$ and $I_{\tilde{\mathcal{E}}_{n}}$ live in a smaller-dimensional space than those defined by $I_{\mathcal{Z}_{n}}, I_{\mathcal{C}_{n}}$, and $I_{\mathcal{E}_{n}}$, respectively; but we may embed them in the natural way into a higher-dimensional space. The hyperplanes are defined by the rows indexed by the $\lambda_{i j}$ 's.

In general, multi-homogeneous part of any given ideal is not generated by the multihomogeneous generators of the original ideal. But for the ideal of the $p_{1}$ model we are able to use homogeneity to decompose the Markov moves into "essential" parts. The upshot of this result is that analysis and computations become easier. In addition, all moves obtained this way are applicable and irredundant.

Theorem 3.22 (Essential Markov moves for the $p_{1}$ model). The Markov moves for the $p_{1}$ model in the case of zero and edge-dependent reciprocation can be obtained from the Graver basis of the common submatrix $\mathcal{A}_{n}$, together with the Markov basis for the ideal $Q$ as defined in Theorem 3.15 .

Remark 3.23. The case of constant reciprocation is more challenging as we do not yet have a simple decomposition as in the other two cases. However, a similar argument can be used to claim that the Markov moves in case of constant reciprocation can be obtained by repeating the moves for the simplified model, while respecting the multi-homogeneity requirement.

Before embarking on a proof of 3.22 , let us make the claim more precise. Take $q=$ $q^{+}-q^{-} \in I_{\tilde{\mathcal{Z}}_{n}}$ or $I_{\tilde{\mathcal{E}}_{n}}$ in the ideal of the simplified model. The monomials $q^{+}$and $q^{-}$are represented by directed edges in our $n$-node network. If $q^{+}$and $q^{-}$represent the same cycle with different orientation, then $q$ is multi-homogeneous already. On the other hand, suppose

$$
q=\prod_{k=1}^{d} p_{i_{k} j_{k}}\left(a_{k}, b_{k}\right)-\prod_{k=1}^{d} p_{s_{k} t_{k}}\left(c_{k}, d_{k}\right)
$$

where $p_{i_{k} j_{k}}\left(a_{k}, b_{k}\right), p_{s_{k} t_{k}}\left(c_{k}, d_{k}\right) \in\left\{p_{i j}(1,0), p_{i j}(0,1), p_{i j}(1,1)\right\}, i<j$, for $1 \leq k \leq d$. Then we may define

$$
\tilde{q}:=\prod_{k=1}^{d} p_{i_{k} j_{k}}\left(a_{k}, b_{k}\right) \prod_{k=1}^{d} p_{s_{k} t_{k}}(0,0)-\prod_{k=1}^{d} p_{s_{k} t_{k}}\left(c_{k}, d_{k}\right) \prod_{k=1}^{d} p_{i_{k} j_{k}}(0,0) .
$$

Also, one can modifiy $\tilde{q}$ by taking $k$ in a subset of $\{1, \ldots, d\}$, as we may not need every $k$ from 1 to $d$ to make $q$ multi-homogeneous. We call each such $\tilde{q}$ a lifting of $q$, including the 
case when $q$ is lifted using less then $d$ variables in each term. (Note: in addition, if not all of $\left(a_{k}, b_{k}\right)$ and $\left(c_{k}, d_{k}\right)$ are $(1,1)$, we may lift $q$ by $p_{i j}(1,1)$ instead of $p_{i j}(0,0)$.)

It is clear that all of these lifts are in the toric ideal of the model. It is not clear that these are sufficient to generate it (or give its Gröbner basis). In particular, it seems that there is another kind of lifting that needs to be included to generate the ideal of the model. Essentially, it involves overlapping two (or more!) minimal generators of the ideal $I_{\mathcal{A}_{n}}$ in a special way.

For example, consider the binomial

$$
p_{12}(1,0) p_{13}(0,1) p_{14}(0,1) p_{23}(1,1)-p_{12}(0,1) p_{13}(1,1) p_{23}(0,1) p_{24}(0,1)
$$

which is in the ideal for the model on $n \geq 4$ nodes. It is not of the form $\tilde{q}$, that is, it is not lifted in the nice way described above. However, it can still be obtained from binomials on the graph on 3 nodes. Let $f^{+}=p_{12}(1,0) p_{13}(0,1) p_{23}(1,0), f^{-}=p_{12}(0,1) p_{13}(1,0) p_{23}(0,1)$ and $g^{+}=p_{14}(0,1) p_{23}(0,1), g^{-}=p_{13}(0,1) p_{24}(0,1)$. Note that $f=f^{+}-f^{-}$and $g=g^{+}-g^{-}$ are in the simplified model ideal for $n \geq 3$ nodes. If we use the fact that in the graph the edges from node 1 to node 3 and from node 3 to node 1 combine to a double-ended edge between 1 and 3 , and if we define $f \otimes g=f^{+} g^{+}-f^{-} g^{-}$, then we obtain precisely $p_{1,2}(1,0) p_{1,3}(0,1) p_{1,4}(0,1) p_{2,3}(1,1)-p_{1,2}(0,1) p_{1,3}(1,1) p_{2,3}(0,1) p_{2,4}(0,1)$. We will call such an operation $(f \otimes g)$ an "overlap" of two binomials, since it corresponds to overlapping the edges of the graphical representations of $f^{+}-f^{-}$and $g^{+}-g^{-}$. Take a binomial $f \otimes g$ in the ideal of the model $I_{\mathcal{E}_{n}}$. Note that it may happen that neither $f$ nor $g$ are in $I_{\mathcal{E}_{n}}$. But in terms of moves, $f \otimes g$ is equivalent to performing two successive moves: the one defined by $f$, and the one defined by $g$. In particular, binomial overlaps give rise to consecutive Markov moves which respect multi-homogeneity.

Remark 3.24. Note that $Q$ appears only in the decomposition for the ideal $I_{\tilde{\mathcal{E}}_{n}}$, and not $I_{\tilde{\mathcal{Z}}_{n}}$. But for example the binomial $p_{12}(1,1) p_{34}(1,1) p_{23}(0,0) p_{14}(0,0)-p_{12}(0,0) p_{34}(0,0) p_{23}(1,1) p_{14}(1,1)$ is a homogenization of a generator of $Q$, and it lives in the ideal $I_{\tilde{\mathcal{Z}}_{4}}$. Homogenization by $p_{i j}(0,0)$ does not affect the move itself.

Proof of Theorem 3.22. Clearly, all moves from $I_{\mathcal{A}_{n}}$ and $Q$ can be homogenized by lifting: that is, if $q \in I_{\mathcal{A}_{n}}$, then $\tilde{q} \in I_{\mathcal{E}_{n}}$. The proof relies on a simple, yet crucial, observation that by definition, the ideal of the model is contained in the ideal of the simplified model; e.g. $I_{\mathcal{E}_{n}} \subset I_{\tilde{\mathcal{E}}_{n}}$.

Let $f \in I_{\mathcal{E}_{n}}$. Then $f \in I_{\tilde{\mathcal{E}}_{n}}$. If $f$ is in the Graver basis of $I_{\tilde{\mathcal{E}}_{n}}$, then we are conclude by Theorem 3.18. Alternately, assume $f$ is not in the Graver basis of $I_{\tilde{\mathcal{E}}_{n}}$. Then there exists a binomial $p \in I_{\tilde{\mathcal{E}}_{n}}$ such that $p^{+}$divides $f^{+}$and $p^{-}$divides $f^{-}$. Equivalently, we can write $f$ as $f=p \bigotimes \tilde{f}$ for $\tilde{f}$ defined appropriately (e.g. $\tilde{f}^{+}:=f^{+} / p^{+}$). Since we may assume that $p$ is primitive, we can keep decomposing $\tilde{f}$ until we obtain an overlap of $k$ primitive binomials from the ideal of the simplified model. Note also that we may assume that in the end we are not in the case where $\tilde{f}^{+}=\tilde{f}^{-}$. Indeed, if $f$ is a multiple of another binomial in the ideal, say, $f=\tilde{f}^{+} g^{+}-\tilde{f}^{+} g^{-}$, then $g^{+}-g^{-}$is also in the ideal. In terms of moves, multiples do not contribute anything: they instruct us to remove and add the same edge.

Replacing $\mathcal{E}_{n}$ by $\mathcal{Z}_{n}$ does not change the above argument.

Using these two constructions of lifting and overlapping, we make the following conjecture: 
Conjecture 3.25. Minimal Markov (Gröbner) bases for the $p_{1}$ models can be obtained from Markov (Gröbner) bases of $I_{\mathcal{A}_{n}}$ by repeated lifting and overlapping of the binomials in the minimal Markov bases of various $(n-1)$-node subnetworks.

We close this section by remarking that these various liftings imply a (possibly non-sharp) degree bound on the Markov and Gröbner bases for the model. For example, each lifting of the first stype will add at most $\left(\begin{array}{l}n \\ 2\end{array}\right)$ edges to each monomial of $q$ thus increasing the degree by at most $\left(\begin{array}{l}n \\ 2\end{array}\right)$ from the degree needed for the simplified model, while overlapping $k$ binomials will allow the degrees of generators to increase $k$ times. Note that we have already seen lifts and overlaps in Examples 3.2 and 3.3 .

\section{Discussion}

We close with a discussion about the relationship between our parametrization and the loglinear parametrization suggested by $[9,10$. Fienberg and Wasserman's parametrization of the $p_{1}$ model encodes it as a $n^{2} \times 2 \times 2$ contingency table, where the first variable corresponds to a dyad and the second and third represent the four dyadic configurations. Thus, a network is represented as a point $x$ in $\mathbb{R}^{4 n^{2}}$ with $0 / 1$ entries. This log-linear parametrization is clearly highly redundant, as, besides the Multinomial constraints on each of the $n^{2}$ dyads, there are additional symmetric constraints of the form $x_{i, j, k, l}=x_{j, i, k, l}$, for all $i, j \in\{1, \ldots, n\}$ and $k, l \in$ $\{0,1\}$. Although, as shown in [11], these redundancies can be convenient when computing the MLE, they are highly undesirable for finding Markov bases. Indeed, the toric ideal corresponding to the this parametrization has $4 n^{2}$ indeterminates while our parametrization only contains $2 n(n-1)$. For example, when $n=5$, this means the toric ideal lives in the polynomial ring with 100 indeterminates, instead of 40 that we have. In addition, the number of generators explodes combinatorially: for the case of constant reciprocation, $\rho_{i j}=\rho$, the ideal of the network on $n=3$ nodes has 107 minimal generators, and the one of the 4-node network has 80,610 . The case when $n=5$ is hopeless: even 4 ti2, the fastest software available to compute generating sets of toric ideals, cannot handle it. One wonders what all of these extra generators are! First of all, note that this contingency-table representation is highly redundant. Most of the Markov basis elements are inapplicable because of the product multinomial constraints and the symmetry constraints. Finally, the many symmetries in the table imply many highly non-trivial constraints that have to be accounted for when eliminating non-applicable moves. We were able to analyze the $n=4$ case and reduce all of the 80610 moves to the ones we get using the design matrices $\mathcal{C}_{n}$, but the effort was nontrivial. Therefore, at least from the point of view of studying Markov bases, the parametrization we are using in the present paper is preferable.

Our hope is that this article motivates a deeper study of the algebraic statistical challenges for $p_{1}$ models and their extensions.

\section{REFERENCES}

[1] 4ti2 team: 4ti2 - A software package for algebraic, geometric and combinatorial problems on linear spaces. Available at www.4ti2.de.

[2] Y.M. Bishop, S.E. Fienberg and P.W. Holland (1975). Discrete Multivariate Analysis: Theory and Practice, MIT Press. Reprinted by Springer, 2007. 
[3] B. Buchberger (1976). "Theoretical Basis for the Reduction of Polynomials to Canonical Forms," SIGSAM Bull., 39, 19-24.

[4] A. Corso and U. Nagel (2009). "Monomial and toric ideals associated to Ferrers graphs", Trans. Amer. Math. Soc. 361, 1371-1395.

[5] H. Ohsugi and T. Hibi (1999). "Toric Ideals Generated by Quadratic Binomials," Journal of Algebra, 218 (2), 509-527.

[6] D. Cox, J. Little and D. O'Shea (2005). Using Algebraic Geometry, Graduate Texts in Mathematics 185, Springer-Verlag, Berlin.

[7] P. Diaconis and B. Sturmfels (1998). "Algebraic algorithms for sampling from conditional distribution." Annals of Statistics, 26, 363-397.

[8] M. Drton, B. Sturmfels and S. Sullivant (2009). Lectures in Algebraic Statistics. Oberwolfach Seminars, Birkhauser.

[9] S.E. Fienberg and S.S. Wasserman (1981). "Categorical Data Analysis of Single Sociometric Relations," Sociological Methodology 1981, 156-192.

[10] S.E. Fienberg and S.S. Wasserman (1981). Discussion of Holland, P. W. and Leinhardt, S. "An Exponential Family of Probability Distributions for Directed Graphs," Journal of the American Statistical Association, 76, 54-57.

[11] S.E. Fienberg, M.M. Meyer and S.S. Wasserman (1985). "Statistical Analysis of Multiple Sociometric Relations," Journal of the American Statistical Association, 80 (1985), 51-67.

[12] A. Goldenberg, A.X. Zheng, S.E. Fienberg and E.M. Airoldi (2009). "A Survey of Statistical Network Models," Foundations and Trends in Machine Learning, to appear.

[13] S.J. Haberman (1981). Discussion of Holland, P. W. and Leinhardt, S. "An Exponential Family of Probability Distributions for Directed Graphs," Journal of the American Statistical Association, 76(373), $60-61$.

[14] H. Hochster (1972). "Rings of invariants of tori, Cohen-Macaulay rings generated by monomials, and polytopes," Annals of Mathematics, 96, 318-337.

[15] P.W. Holland and S. Leinhardt (1981). "An Exponential Family of Probability Distributions for Directed Graphs (With Discussion)," Journal of the American Statistical Association, 76, 33-65.

[16] S. Lauritzen (1996). Graphical Models, Oxford University Press.

[17] G. Pistone, E. Riccomagno, E. and H.P. Wynn (2001). Algebraic Statistics: Computational Commutative Algebra in Statistics, Chapman \& Hall, New York.

[18] A. Rinaldo, S. Petrović and S.E. Fienberg (2009). " Maximum Likelihood Estimates for a Directed Random Graph Model with Reciprocation", in preparation.

[19] B. Sturmfels (1996). Gröbner Bases and Convex Polytopes. American Mathematical Society, University Lecture Series, Vol. 8.

[20] R. Villarreal (2001). Monomial Algebras. Monographs and Textbooks in Pure and Applied Mathematics, Vol. 238. Marcel Dekker, Inc., New York.

Department of Mathematics, Statistics, and Computer Science, University of Illinois, Chicago, IL, 60607

E-mail address: petrovic@math.uic.edu

Department of Statistics, Carnegie Mellon University, Pittsburgh, PA 15213

E-mail address: arinaldo@stat.cmu.edu

Department of Statistics and Machine Learning Department, Carnegie Mellon UniverSity, PitTsburgh, PA 15213

E-mail address: fienberg@stat.cmu.edu 